\title{
Universals and diversity in gesture: Research past, present, and future
}

\author{
Kensy Cooperrider \\ kensycoop@gmail.com
}

\begin{abstract}
At the dawn of anthropology, gesture was widely considered a "universal language." In the $20^{\text {th }}$ century, however, this framing fell out of favor as anthropologists rejected universalism in favor of relativism. These polemical positions were largely fueled by high-flying rhetoric and second-hand report; researchers had neither the data nor the conceptual frameworks to stake out substantive positions. Today we have much more data, but our frameworks remain underdeveloped and often implicit. Here, I outline several emerging conceptual tools that help us make sense of universals and diversity in gesture. I then sketch the state of our knowledge about a handful of gestural phenomena, further developing these conceptual tools on the way. This brief survey underscores a clear conclusion: gesture is unmistakably similar around the world while also being broadly diverse. Our task ahead is to put polemics aside and explore this duality systematically — and soon, before gestural diversity dwindles further.
\end{abstract}

keywords: gesture, human diversity, human universals, anthropology, communication, culture

* Note: This is the accepted version of an article in press; it does not include minor changes that may result from copy-editing. Please quote only the final published version. 
UNIVERSALS \& DIVERSITY IN GESTURE

\section{Introduction}

Is gesture universal? Is it part of our built-in bodily know-how, the birthright of our species? Or is it variable, learned through exposure to the quirks and conventions of our community? Or could it be both-universal in some respects and variable in others? To grapple with these questions is to grapple with some of the most contentious ideas in the Western tradition - about what is innate and what is learned, about what is biological and what is cultural, about what is natural and what is conventional. It is perhaps unsurprising, then, that scholars have circled questions about universals and diversity in gesture for centuries, and a cynical observer might guess we are destined to circle them for centuries more. I take a rosier view: I think we are poised to learn a lot about how gesture does and does not vary around the world. But we have to get moving. As I will discuss later, humanity's rich gestural diversity appears to be dwindling.

But before turning to the future, it is worth taking stock of the past. Prior to the $20^{\text {th }}$ century, questions about gestural diversity often took the form of the question: Is gesture a universal language? For centuries, the consensus answer was a barely qualified yes (for discussion, see: Carayon, 2016; Cooperrider, 2018; Kendon, 1984, 2004;

Knowlson, 1965; Knox, 1990). One of the earliest universalist pronouncements was made by Quintillian, the Roman rhetorician, who wrote that "though the peoples and nations of the earth speak a multitude of tongues, they share in common the universal language of the hands" (Quintillian, 1922 [orig. 95 C.E.], Book XI, III, 87). Perhaps the fullest—and most florid — endorsement of the "gesture as universal language" idea came centuries later, in the work of John Bulwer, an English physician. In a 1644 treatise, he wrote that gesture is the "tongue and general language of human nature which, without teaching, 
men in all regions of the habitable world do at first sight most easily understand" (Bulwer, 1975 [orig. 1644], p. 16). Gestures, he continued, "had the happiness to escape the confusion at Babel” (p. 16) (see Kendon, 1984).

A universalist stance toward gesture held sway into the early days of anthropology in the $19^{\text {th }}$ century, blending in with a general universalist bent of the times. In one proto-anthropological treatment, Joseph Marie Dégerando (1969 [orig. 1800]) described gestures as "the signs that were closest to nature" (p. 71), and he urged travelers to document them. Half a century later, Edward B. Tylor, a founding figure in the field, took a keen interest in gestural communication and other visual forms of expression. He wrote that gestures could be understood "without the aid of history, as direct products of the human mind" (Tylor, 1878, p. 3). Around the same time, Garrick Mallery, known for his work on the sign language systems of native North America, also struck a universalist chord. He argued that the bodily communication systems of Native Americans, of the deaf, and, indeed, of all peoples "together constitute one language - the gesture speech of mankind—of which each system is a dialect" (Mallery, 1882, p. 76). To the extent that such universalist positions were based on evidence, they were often based on two observations. The first was that, during first contacts between European explorers and indigenous peoples, gestural communication was often successful in a way that verbal communication was not (Hewes, 1974; see also Bonvillian, Ingram, \& McCleary, 2009; Vandenabeele, 2002). The second observation was that deaf people were able to create sophisticated communication systems with their hands, as if the gestural medium came to them naturally. Both observations are accurate enough; whether they show that gesture is "a universal language" is another matter. 
Beginning in the $20^{\text {th }}$ century, the universalist stance of early anthropology began to retreat, and a reflexive relativism took hold (Engelke, 2018; Stocking, 1995). Gesture largely seems to have fallen out of favor as a topic of inquiry during this time (Kendon, 2004), but commentators still found occasion to remark on it. Edward Sapir wrote of a "secret code" of gesture, which he considered "artificial" and "as definitely a creation of social tradition, as language or religion or industrial technology" (Sapir, 1927, p. 556). In an influential paper, Marcel Mauss made the case that much mundane bodily behaviorwalking, running, swimming, descending stairs—was culturally inflected (Mauss, 1973 [orig. 1935]). He did not discuss communicative gestures per se, but his treatment modeled how the emerging relativism of the times could be extended to bodily behavior. In another triumph for relativism, David Efron showed that one's gestural style, including both the quantity and quality of one's gestures, was not determined by "racial descent," as had been claimed in the Nazi era, but by one's socio-cultural environment (Efron, 1941). In short, gesture was malleable, not fixed.

Over this history, universalist and relativist positions were rarely unalloyed. Commentators often recognized both the universal and variable dimensions of gesture, even while trumpeting one or the other side of this duality. A common rhetorical move was - and remains - to deny the purity of the opposing side rather than assert the purity of one's own. The universalist would acknowledge the existence of conventions in gesture, but quickly assert that gesture was no "mere" product of culture; the relativist might concede the importance of biology in shaping communicative behavior, but then quickly deny that gesture was entirely "determined" by biology. Sapir (1927/1949), for instance, characterized gesture as "by no means referable to simply organic responses" 
(p. 556). These positions had something else in common: a lack of systematic evidence. With a few exceptions - notably Efron—-the tools of argument were soaring rhetoric, second-hand report, and appeal to intuition. Gesture was treated with a broad, totalizing brush; claims about gestural communication as a whole were often made on the basis of thin descriptions of isolated phenomena.

How to proceed? To make real progress - to build a subfield devoted to the systematic study of universals and diversity in gesture - we need two things. First, we need better data. We can't afford to overgeneralize on the basis of thin, scattershot descriptions. We need data from culturally and geographically disparate communities, and we need such data to be systematically collected for comparative purposes. And yet data on its own is not enough. The second thing we need is better, more explicit conceptual frameworks. That is, we need intellectual tools to help us make sense of the data we already have, as well as to guide the collection of more data. Valuable conceptual tools have recently emerged in the field, and a goal of the present paper is to help crystallize and consolidate these.

A brief historical case study will help to illustrate this need for better data and better conceptual tools. A phenomenon of particular fascination in the early days of the anthropology of gesture was bodily signals for negation and affirmation. Such signals are naturally among the first that travelers will notice: it is hard to get far into any social interaction until you understand what your interlocutors are affirming and what they are denying. By looking more closely at how this class of gestures has been treated, we can see weak points in how questions about gestural diversity have been posed and answered. 
UNIVERSALS \& DIVERSITY IN GESTURE

\section{A case study: Negation and affirmation}

An insightful early treatment of signals for negation and affirmation can be found in Darwin's Expression of Emotions in Man and the Animals (1872). Much of Darwin's discussion is based on observations he solicited by correspondence from acquaintances in distant lands. He set out to demonstrate the universality of the head shakes and nods familiar to his British readers, but he ended up concluding that such signals were "not so universally employed as I should have expected" (Darwin, 1872, p. 274). He notes, for instance, examples where negation is reportedly done with the hands rather than the head (Table 1). According to one correspondent, in the Torres Strait of northern Australia, negation is done by raising a hand and "shak[ing] it by turning it round and back two or three times" (p. 275). Overall, Darwin observes "considerable diversity" (p. 277) in these signals, but also "much uniformity" (p. 277), and he concludes that "they seem too general to be ranked as altogether conventional or artificial" (p. 274). He accounts for this uniformity by describing how several of the most prominent negation signals - the head shake and the backward jerk—-may be rooted in acts of refusing food in infancy.

Table 1. Gestures for negation reported in Darwin (1872)

\begin{tabular}{ll}
\hline gesture & region or group \\
\hline lateral head shake & Anglo-Saxon, Malaysia, Ceylon (Sri \\
& Lanka), China, coastal Guinea, \\
& Australia, Fuegian (Argentina) \\
backward head jerk with tongue protrusion & Gipp's Land (Australia) \\
backward head jerk with tongue cluck & Greece, Turkey \\
backward head jerk to side with tongue cluck & Hindoo (India) \\
head jerk to shoulder with tongue cluck & Abysinnia (Ethiopia)
\end{tabular}


UNIVERSALS \& DIVERSITY IN GESTURE

squinting of eyes

winking of eyes

one hand held and rotated several times

open hand moved outwards, palm facing in

finger or whole hand shaken side to side
Dyaks (Borneo)

Esquimaux

Torres Strait (Australia)

Indians of North America

Indians of North America, Italian, Anglo Saxon

Note: The table retains Darwin's labels and spellings of regions/groups, though in some cases these are no longer used.

Other scholars have been less balanced in their interpretations. In a paper titled 'The cultural basis of emotions and gestures,' Weston LaBarre (1947), working within the anti-universalist lather of $20^{\text {th }}$ century anthropology, used very similar observations to emphasize the non-universality of signals for negation and affirmation. He writes that "a rocking of the skull forward and backward upon its condyles, which rest on the atlas vertebra, as an indication of affirmation and a rotation on the axis vertebra for negation... [are] by no means widespread ethnologically" (p. 49-50). He supports this generalization with examples of other attested signals. The Ainu of Japan, for instance, negate by passing the hand "from right to left and back in front of the chest" (p. 50); people in Calcutta affirm by drawing the head "rapidly in an arc from shoulder to shoulder, usually four times" (p. 50). LaBarre's relativist position appears to rest on a slippage between the claim that head shakes and nods are not universal—which is inarguable — and the claim that they are "by no means widespread"- which is questionable, as Darwin showed.

A third treatment — more balanced than LaBarre's — is found in Roman Jakobson's squib, 'Motor signs for yes and no' (1972). He sketches three different 
systems that illustrate the diversity of head signals for negation and affirmation found even within Europe. A first system, prevailing in Western Europe, involves a side-to-side shake for 'no' and an up-and-down nod for 'yes.' A second, found in Greece, Turkey, and other parts of the Mediterranean, involves a backward jerk for 'no' and an up-anddown nod for 'yes.' A third, found in Bulgaria, involves a side-to-side movement for affirmation and an up-and-down movement for negation. Jakobson, like Darwin, resists the possibility that such signals are "purely arbitrary convention" (p. 92); and, like Darwin, he sees the topic as rich ground for further inquiry. He closes by urging a systematic investigation of how signals for negation and affirmation exhibit an “interrelation of naturalness and conventionality" (p. 95).

Perhaps the clearest lesson that can be drawn from these early observations is that we need better data, in a few senses. First, we simply need better characterizations of the behaviors of interest. Brief verbal descriptions of the type found in such early sources are rarely adequate for the task of systematic comparison. Is the Ainu pattern noted by LaBarre a manual echo of a side-to-side head shake? It's hard to tell. (The prevalence of manual signals for negation that echo head signals was noted already by Darwin [see Table 1]). Is the Bulgarian system described by Jakobson really a "striking diametrical opposition" of the shake-for-no, nod-for-yes pattern (Jakobson, 1972, p. 91)? It's unclear. Second, for many types of claims, we need systematically sampled data. One way to do this is to use a systematic procedure for eliciting the behavior of interest, such as a referential communication task; another approach is to use a systematic procedure for selecting examples from naturalistic data, as is currently done to good effect in corpusbased studies of conversation (e.g., Dingemanse et al., 2015; San Roque et al., 2015). 
Systematic sampling across cultures is another matter, with many attendant challengesespecially the thorny issue of non-independence—-but for now, any comparative studies in which the gestures are carefully elicited or selected are a welcome start.

Another clear lesson is that we need observations, not just about isolated gestures like the head shake, but about broader gestural repertoires. For basic communicative functions like negation, a community will often have a rich set of conventional signals. Anglophone and European speakers, for instance, negate with head shakes, certainly, but also with a suite of manual gestures (e.g., Harrison, 2014). Indeed, the few thorough studies of gestural negation to date suggest that many—perhaps most—communities have similarly rich repertoires (e.g., Inbar \& Shor, 2019; Mesh \& Hou, 2018). Once we recognize such richness as the norm, we realize that the value of reporting a single signal for negation or affirmation—as early observers usually did—is limited. The negation gesture that Darwin ascribes to the Torres Strait (or one similar to it) is also used by the Yupno of Papua New Guinea-but, in addition to this gesture, Yupno speakers may shake their heads or pout their lips to negate. The side-to-side assent gesture used in Calcutta and mentioned by LaBarre is still in use across India, as far as can be gleaned from YouTube videos ${ }^{1}$ — but, critically, so are head nods.

Another major issue concerns generalizability. What is the value of observations about specific classes of gesture, such as negation, when our goal is to draw conclusions about gestural universals and diversity generally? After all, some gestural phenomena may exhibit more diversity than others. Interestingly, universalist treatments of gesture

\footnotetext{
${ }^{1}$ Sadly, I am not aware of any scholarly discussions of this gesture, sometimes called the "head bobble." However, several light-hearted treatments are available on YouTube (e.g., https://www.youtube.com/watch?v=Uj56IPJOqWE; accessed February 20, 2020).
} 
for the most part barely grazed over negation (see, e.g., Tylor, 1878). Wiggle room in what phenomena we focus on — and generalize from - means that universalists and relativists can easily talk past each other. Those committed to demonstrating universality can focus on broader gestural techniques like pointing and depiction (e.g., Mallery, 1882); those committed to demonstrating diversity can focus on gestures with clear conventional aspects (e.g., Archer, 1997). And yet both parties can claim that their positions are grounded in facts.

These are just a few of the issues with past research we need to contend with if we are to make progress in our thinking about gestural diversity and universals. The next section turns to the present. I outline several distinctions that have recently emergedconceptual tools that can help us address some of the issues just described. These tools shed light, not only on gestural signals for negation and affirmation, but also on other gestural phenomena, as I'll show later.

\section{Assembling a toolkit}

Here I sketch a few key distinctions that deserve a more prominent place in our conceptual toolkit for thinking about gestural universals and diversity. These distinctions have emerged in recent years but remain somewhat implicit. My aim here is to crystallize and consolidate them.

\subsection{Conventions, kinds, and properties}

If one were asked whether language is universal, a natural response would be 'What aspects of language?' And so it should be with gesture. On the one hand, the 
capacity to gesture - perhaps the urge to do so — is universal (Kendon, 2004; Kita, 2009; McNeill, 1992). On the other, there are many particular gestures - that is, specific mappings between gesture form and meaning - that are far from universal (Archer, 1997; Morris, Collett, Marsh, \& O’Schaughnessy, 1979). As already noted, relativists can thus point to kaleidoscopic variation in specific form-meaning pairings as evidence for striking diversity; universalists can reasonably counter by emphasizing broadly shared general techniques as evidence for overwhelming uniformity (Kendon, 1984). To break this dynamic, it is helpful to use more finely differentiated vocabulary.

Gestural phenomena can be characterized at different levels of abstraction. At a low level of abstraction, we have relatively constrained mappings between specific meanings and specific forms - that is, specific gestures (Figure 1). At a higher level, we have relatively unconstrained mappings between broad classes of meanings and broad classes of forms - that is, general kinds of gestures. For simplicity, I'll refer to the lower level as gestural conventions and the higher level as gestural kinds. By gestural conventions I mean conventional pairings of form and meaning — as in the everyday sense of a "gesture for negation," a "gesture for crazy," and so on. The gestures that fit this description are often called "emblems" or "quotable gestures" (see, e.g., Brookes, 2004; Johnson, Ekman, \& Friesen, 1975; Kendon, 2004; McNeill, 1992). In addition to familiar examples of emblems - the thumbs up, the shhh-gesture, the peace sign — this lower level includes conventional forms used in greeting, beckoning, conveying numbers, and so on. I would also include here "gesture families" (Kendon, 2004; Müller, 2004) and “recurrent gestures” (Bressem \& Müller, 2014; Ladewig, 2014; Müller, 2017). Both are 
broader in scope than emblems - that is, they comprise a wider range of forms and a wider range of meanings - but are still relatively constrained.

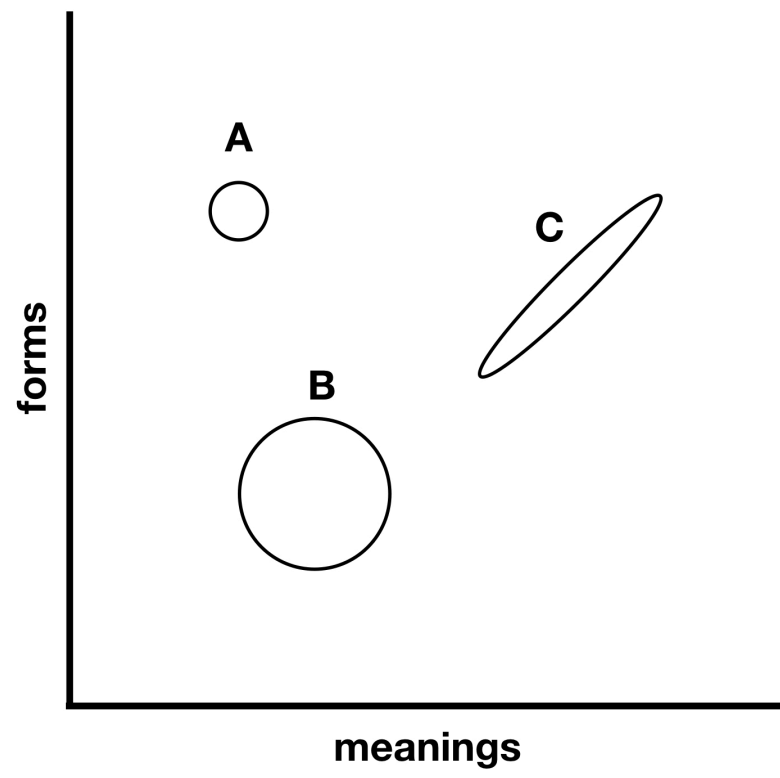

Figure 1. A schematic depiction of different types of gestural conventions. In a muchsimplified space of all gestural forms and all gestural meanings, different types of gestural conventions have different "shapes." Emblems - marked A-involve a narrow mapping between form and meaning. Gesture families and recurrent gestures-marked $\mathrm{B}$ - involve a broader range of interrelated forms and meanings. Gestural practicesmarked $\mathrm{C}$-involve a parameter of form that maps on to a parameter of meaning.

Still within this lower-level category of gestural conventions there is another class, largely ignored to date, of what I term gestural practices. In gestural practices what is conventionalized is a gradable parameter of form that maps onto a gradable parameter of meaning. An example is the practice of pointing with exaggerated height to refer to distant entities, as found in Mesoamerica and elsewhere (Mesh, 2017; see also Le Guen, 2011a). In this case, a gradable parameter of form — gesture height—maps on to a gradable parameter of meaning - referent distance. Other examples include the use of socalled "measuring gestures," best described in Africa (Nyst, 2016), and the convention of 
pointing to the sun's east-west arc to indicate the time of day (Floyd, 2016; Le Guen \& Pool Balam, 2012).

The higher level consists of gestural kinds. I refer to this as a higher level of abstraction because, whereas gestural conventions refer to specific form-meaning pairings (as in emblems) or circumscribed clusters of form-meaning pairs (as in gesture families, recurrent gestures, and gesture practices), gestural kinds cover dispersed swaths of form and meaning. Such kinds include, for instance, the members of McNeill's (1992) typology—beats, iconics, metaphorics, deictics, and emblems — and the gestures that exemplify Clark's $(1996,2016)$ typology of methods of communication —indicating, symbolizing, and depicting. Both typologies are rooted in Peircean semiotics, and encompass what we might call general semiotic techniques (Enfield, 2009; Kendon, 1984). Within these broad techniqes, we also have subtechniques, such as enacting, tracing, and other types of iconicity (e.g., Kendon, 2004; Mandel, 1977). The general technique of indicating also includes several subtechniques, including pointing, touching, and brandishing (Clark, 2003). Other gesture types that sit at the higher level include Bavelas and colleagues' notion of topic versus interactive gestures (Bavelas, Chovil, Lawrie, \& Wade, 1992), as well as my own distinction between foreground and background gestures (Cooperrider, 2017).

Finally, we may also consider general properties of gesture. These include, for instance, the observation that gesture and speech are tightly co-timed (McNeill, 1992; Pouw, Harrison, \& Dixon, 2019); that gesture and speech are semantically co-expressive (McNeill, 1992; Schegloff, 1984); that gesture strokes are much more likely to anticipate the speech they go along with than to lag behind it (e.g., Nobe, 2000); that gesture is 
usually synchronized with stressed elements of speech (e.g., Brentari, Marotta, Margherita, \& Ott, 2013); that one-handed gestures are usually produced with the dominant hand (McNeill, 1992); and so on.

How does the three-way distinction between gestural conventions, kinds, and properties help with questions about universals and diversity? For starters, gestural kinds — or at least those proposed to date — are all presumed to be universal. It would be truly shocking if a community were discovered in which gestures were never used to depict; or in which some part of the body was not used to point; or in which emblematic gestures were altogether absent. This would be shocking because these phenomena seem to spring from basic cognitive biases, from hunches that humans all share about how to use the body meaningfully. Of course, there is still much variability in precisely how people depict, indicate, symbolize, and so on. This variability stems from differences in underlying cognitive models, different communicative ideologies, different linguistic structures, and perhaps different communicative ecologies, as discussed later (Kita, 2009). But the kinds themselves are universal. Gestural properties are also widely presumed to be universal, though this is an empirical question (see, e.g., de Ruiter \& Wilkins, 1998).

Gestural conventions are another matter, and this is where many of the most interesting questions about gestural diversity reside. The most narrowly scoped gestures - emblems - appear to vary widely. In certain cases, for instance, the same form is paired with radically different meanings - e.g., the thumbs-up in the United States versus in Iran (Archer, 1997). But as soon as we move up a notch in abstraction, to more broadly scoped gestures — gesture families, recurrent gestures, and gestural practices—we 
begin to see striking similarities across cultures. For example, several prominent recurrent gestures recur across groups (e.g., Bressem, Stein, \& Wegener, 2017); the palmup epistemic gesture is a broadly shared pairing of form and meaning (Cooperrider, Abner, \& Goldin-Meadow, 2018); and many conventional negation gestures are strikingly widespread (see, e.g., Inbar \& Shor, 2019; Mesh \& Hou, 2019). We might say, borrowing Darwin's words, that these gestures seem too widespread to be thought of as "altogether conventional or artificial" (Darwin, 1872, p. 274). I argue later that they are best considered "natural conventions."

\subsection{Presence and privilege}

A second emerging conceptual tool concerns the distributional questions we are interested in. Most basically, we might be interested in which gestures are attested in which communities - that is, we might be interested in questions about gesture presence or absence. We might want to know which meanings have conventional gestures associated with them; or, for meanings that seem to be universally enshrined in gesture (e.g., beckoning), which form is used in a given community. Issues like these are what many think of when they first think of gestural universals and diversity. But for some of our most basic gestures, there is more than one form that serves the same function: there is more than one way to point, more than one way negate, more than one way to depict size or motion. Thus, we might also be interested in questions, not just about which gestures are present, but about which are privileged.

A gesture is privileged, as I use a term, if it is more culturally prominent or important. There are at least three different ways that such privilege manifests. A first 
way is preference - a gesture is privileged if it is used more often, in terms of sheer frequency, than other options that are available within the community. A second way privilege manifests is prototypicality —a gesture is privileged if it is the one people most strongly associate with the function. A third is developmental primacy-a gesture is privileged if children use it earlier than other options that serve the same function. It seems probable that whichever form is used most often is also regarded as prototypical and is also the first that children master. But, in principle, these different manifestations of privilege could dissociate.

Questions about gesture presence are easier to answer than questions about privilege. To claim that a gesture is present, you need to spot it once (or, preferably, a handful of times). To claim that a gesture is privileged, you need a systematic elicitation or sampling procedure (for questions about preference), a meaningful way of eliciting meta-communicative judgments (for questions about prototypicality), or a way of sampling the behavior in young children (for questions about developmental primacy). Of course, researchers often make claims about privilege based on impressions alone (discussed in Cooperrider, Slotta, \& Núñez, 2018). This is fine as a first approximation, but, to make real progress on these kinds of distributional questions, a more systematic approach is needed.

The distinction between presence and privilege helps shed light on the case of negation and affirmation. Darwin, for all his insightfulness, did not seem attuned to the fact that cultures often have repertoires of negation gestures. Thus, he reports examples of manual negation gestures as though they directly falsify his idea that head shakes and nods might be universal. But the mere presence of a manual form of negation cannot do 
this. To falsify the possibility that head shakes and nods are universally present, we would need a richer characterization of the larger repertoire of forms available for negation. To falsify the possibility that head shakes and nods are universally privileged, we would need to assess one or more of the different ways privilege manifests. After we fine-tune our claims about universality along these lines, we see that Darwin's hoped-for generalization may yet prove correct ${ }^{2}$.

\subsection{Core and extensions}

Much as the same function is often served by different gestural forms, the same form often serves different functions. In accounts of gestures with several meanings, there is often implicitly assumed to be a core meaning, which gets extended through processes akin to semantic/pragmatic extension in spoken language (e.g., see Jurafsky, 1996; Sweetser, 1990). The result is what has been called a meaning network with a coreand-extension structure (Cooperrider, Abner, \& Goldin-Meadow, 2018). One of the first studies to highlight the fact that conventional gestures often have a number of interrelated meanings was Kendon's (2002) examination of head shakes. Though he does not use the terminology of core and extension, Kendon catalogued eight different uses of the headshake, including as an accompaniment to a verbal 'no' and, less obviously, as an intensifier (see also McClave, Kim, Tamer, \& Mileff, 2007). More recently, meaning network approaches have been used to shed light on palm-up gestures (e.g., Cooperrider, Abner, \& Goldin-Meadow, 2018) and size gestures (e.g., Cooperrider \& Núñez, 2012).

\footnotetext{
${ }^{2}$ I am aware of one claim that would seem to undercut the generalization: Turkana speakers reportedly do not use head gestures for negation and affirmation (McClave, 2007). But, unfortunately, I am not aware of any detailed accounts of how Turkana speakers $d o$ negate and affirm.
} 
This distinction between core and extensions highlights another sense in which prior observations were limited. That a gesture is sometimes used for negation does not imply that negation is the gesture's core meaning. The shrug, for instance, is described as part of the negation repertoire in some reports (e.g., Inbar \& Shor, 2019), but in no communities does the shrug appear to have negation as its core meaning.

\section{Four further case studies}

The previous section assembled several emerging distinctions that, I argue, belong in our conceptual toolkit for studying gestural universals and diversity. Over the course of discussion, we have also surveyed the state of current knowledge about gestural negation and affirmation. But, as mentioned, we cannot hang our general understanding of gestural universals and diversity on any one class of gestures. In this section, I sketch the current state of our knowledge by considering four additional gestural phenomena: pointing, palm-up gestures, size gestures, and time gestures. These case studies also serve to further illustrate the proposed toolkit in action, as well as to highlight several complementary approaches to the study of gestural variation. Taken together, these sketches underscore a central conclusion: across communities, gesture is both unmistakably similar and broadly diverse.

\subsection{Pointing}

Pointing in some form or another is, by all accounts, universal. It involves projecting an imaginary vector toward a referent by "moving toward" it with some part of the body (Eco, 1976; Enfield, Kita, \& de Ruiter, 2007; Kendon, 2004); it is a sub- 
technique of indicating, the more general semiotic technique through which people attract attention to referents in the world (Clark, 2003). Pointing is thus a gestural kind but also one put to use in particular gestural conventions - for instance, in Anglo-European emblems (e.g., pointing to the wrist to refer to time) and in gestural practices (e.g., pointing to the sun's arc to refer to time of day).

Across communities, pointing varies in a number of ways. First, there is variation in the repertoire of forms of pointing that are used within a community. Humans are opportunistic and flexible pointers; if the need arises, people will point with their fingers, their heads, their eyes, their umbrellas, or even their feet. But there are also conventional ways of pointing with more restricted geographic distribution, such as lip-pointingwhich involves projecting some part of the mouth while re-orienting gaze toward a referent (e.g., Enfield, 2001)—or nose-pointing — which involves scrunching together the face and nose while re-orienting gaze (e.g., Cooperrider \& Núñez, 2012).

Pointing differs not only in which forms are present within a community, but also in which forms are privileged. In most Anglo-European cultures, pointing with the extended index finger appears to be privileged over other types of manual or non-manual pointing. But this may not be the case everywhere. Wilkins (2003) argued that index finger pointing is not the prototypical form of manual pointing in Arrernte, an Australian Aboriginal language. More radically, several ethnographers have claimed that, in certain groups, non-manual pointing with the head and face is preferred over pointing with the hands (e.g., Everett, 2005). My colleagues and I recently tested this possibility in the Yupno, an indigenous group in Papua New Guinea (Cooperrider, Slotta, \& Núñez, 2018). Using a referential communication task, we found that the Yupno use manual pointing 
(including index finger extended and other handshapes) and non-manual pointing (headand nose-pointing) in roughly equal measure. Finally, one study has proposed that manual pointing universally precedes other forms of pointing in child development. Liszkowski, Brown, Callaghan, Takada, \& de Vos (2012) found that, across seven farflung cultural communities, infants began by pointing with their whole hands and soon thereafter came to favor index finger pointing. These findings suggest a possible universal developmental progression, though the strictest test of this possibility would be in a group like the Yupno where adults do not strongly favor manual pointing.

Another possible dimension of variation across groups is in the extended meanings of pointing. Several studies suggest there may be meaning networks surrounding pointing, but these networks have yet to be adequately characterized. For example, Sherzer (1973) describes uses of lip-pointing for interactive rather than referential functions - for instance, pointing to an interlocutor in the course of mocking. Extended functions for index finger pointing are also attested in Anglo-European cultures, such as the use of pointing to show agreement (Healy, 2012).

\subsection{Palm-up gestures}

Another illuminating case study is the palm-up gesture (e.g., Kendon, 2004; Müller, 2004), a consummate "interactive" or "pragmatic" gesture. When we discuss universality and variation in pointing, we are zooming in on a particular function and asking how it is carried out across cultures. When we investigate palm-ups, we are zooming in on a particular gestural form—sometimes called the "palm-up open hand" 
(Müller, 2004) or "open hand supine" (Kendon, 2004) —and asking what functions it serves across communities.

My colleagues and I recently surveyed observations about how palm-ups are used around the world, focusing on a variant in which the hands are laterally spread out from the body (Cooperrider, Abner, \& Goldin-Meadow, 2018; see also Kendon, 2004). We dubbed this gesture the "palm-up epistemic" because it expresses a cluster of meanings built around a core meaning of "absence of knowledge." Extensions from this core include: expressions of uncertainty, interrogatives, hypotheticals, expressions of obviousness, and exclamatives. Disparate as they may seem, these uses of the palm-up epistemic crop up over and over across the world's cultures; and, remarkably, the palmup serves these same functions in sign languages. (Not all these uses have been described in every community where the gesture is attested, but this could be due to unevenness in the descriptions rather than variability in how the gesture is used.) It remains unclear whether the palm-up epistemic is found universally, but it is clear is that it does not have precisely the same prototypical form everywhere. In Syuba, a language of Nepal, it involves a distinctive hand shape with the fingers partly curled in (Gawne, 2018).

Some studies have illuminated palm-ups by taking a form-centered approach (e.g., Müller, 2004); others have taken a function-centered tack, discussing the palm-up as one means of expressing obviousness (Jehoul, Brône, \& Feyaerts, 2017) or uncertainty (Roseano, González, Borràs-Comes, \& Prieto, 2014). Both approaches have advantages and limitations. The limitation of a purely form-centered approach in this case is that the palm-up form is extremely common as a basic parameter of gesture form; it would thus be unrealistic to expect that all gestures involving this form would share some meaning or 
motivation in common. The limitation with a purely function-driven approach is that palm-ups serve several superficially different functions, such as interrogatives and expressions of obviousness. Thus, if we only focus on one of the palm-up's several uses, we may miss out on the broader meaning networks that make the gesture so interesting.

\subsection{Size gestures}

Humans everywhere likely have occasion to gesture about size. It naturally lends itself to ad hoc depiction — as when people exhibit a length between two palms, a size between the thumb and forefinger, or a height between the hand and the ground. Indeed, size is apparently so ripe for gestural representation that several languages have developed demonstratives that are designed specifically to accompany size gestures, such as 'yay' in English (Cooperrider, 2017; on similar demonstratives in Yucatec Maya, see Le Guen, Petatillo, \& Canché, in press). Beyond ad hoc depictions, many communities also have conventional practices for gesturing about size. A well-known example are the height gestures used in Mesoamerica, which show an entity's height from the hand to the ground while also marking the class of entity with different hand shapes (e.g., Fox Tree, 2009). Interestingly, similar "height gesture classifiers" have emerged elsewhere, such as among the Maori (Best, 1924, p. 439) and the Nuer (Huffman, 1931, p. 69-70). Other communities have developed conventional gestural practices for characterizing the length of small objects or entities - tools, fish, pieces of meat, and the like. Such measurementlike gestures are attested in Africa (Nyst, 2016), southern Italy (V. Evola, pers. comm.), Isthmus Zapotec communities in Mexico (M. McComsey, pers. comm.), and may be more widespread. 
Another case of a gestural convention for expressing size is the diminutive facial gesture found in Yupno (Cooperrider \& Núñez, 2012). To produce this gesture, the area around the face and nose is scrunched together (i.e., the same facial action used in nosepointing; for possible links between the two gestures, see Cooperrider \& Núñez, 2012, p. 119-123). Scrunching the face is a way of making it smaller, providing a likely iconic motivation; and, indeed, a similar form-meaning pairing is attested elsewhere, suggesting it is not arbitrary (e.g., Warlpiri; Kendon, 1988). The gesture often piggy-backs on the morphological diminutive in spoken Yupno, but it is also found more generally with expressions of small size and associated concepts. A first analysis suggests the gesturelike its spoken counterpart—may participate in a larger meaning network, with the core meaning being literal size and possible extensions including expressions of exactness and pragmatic hedges (see Jurafsky, 1996 on similar extensions of diminutives crosslinguistically).

The case of size lends itself to another promising way of uncovering universals and variation in gesture: a domain-centered approach, in which one examines the gestural resources used within a broader semantic domain. One could, for instance, examine how a particular community uses gesture to express different size-related notions like height, length, distance, and so on. I am not aware of any such efforts, however, as researchers more often focus on narrower ranges of gestural meaning.

\subsection{Time gestures}

Time is another semantic domain often represented in gesture (Cooperrider, Núñez, \& Sweetser, 2014). People convey several aspects of time with their hands-the 
relative "location" of events in a sequence, the "length" of a time interval, the "speed" of time's passing, and so on. But the particular spatial concepts used — whether, for example, the past is behind the speaker, to the left, above, or downhill—vary from one community to the next. Generally, people gesture about time using whatever spatial frameworks are most familiar within their community. These frameworks are often pervasive in everyday language or in aspects of visual culture, such as reading direction. English speakers, for instance, gesture about the past and future using either a left-right axis $($ past $=$ left, future $=$ right), which is apparently rooted in reading direction, or a frontback axis (past $=$ back, future $=$ front), which is rooted in linguistic expressions (e.g., Casasanto \& Jasmin, 2012; Walker \& Cooperrider, 2016). Mandarin speakers gesture in both of these ways but also use two additional mappings, one on the up-down axis (past= above, future= below), which seems to be driven by the vertical orientation of some Chinese texts, and another on the front-back axis (past= front, future $=$ back), which seems to be driven by certain linguistic expressions (Gu, Zheng, \& Swerts, 2019). The past-in-front mapping, which strikes many Westerners as peculiar, is in fact attested more broadly, found also in Vietnamese (Sullivan \& Bui, 2016) and, most famously, in Aymara (Núñez \& Sweetser, 2006). At least a few links between time and space appear to be present universally (c.f., Sinha, Sinha, Zinken, \& Sampaio, 2011), such as the conflation of 'here' and 'now' and the idea that time passing is motion in space, but some communities appear to gesture about time in less systematic ways than others (Le Guen \& Pool Balam, 2012; Levinson \& Majid, 2013).

Time, like size, is often depicted ad hoc, but communities sometimes develop highly conventional time gestures. For example, in parts of Europe, gestures conveying 
"in the past" or "the day after tomorrow" are done in consistent, emblem-like ways (Calbris, 1990), as are gestures conveying cyclic patterns (Ladewig, 2011). In Yupno, the diminutive gesture described earlier is also used to characterize small time intervals or to add a shading of precision to a temporal reference-e.g., 'right now' instead of 'now' (Cooperrider \& Núñez, 2012). Another notion sometimes gestured about in highly conventional ways is time of day (e.g., Floyd, 2016; Le Guen \& Pool Balam, 2012). The practice involves pointing to locations on the sun's east-to-west arc to communicate specific points in time (e.g., noon), or by sweeping the hand across segments of this arc to communicate intervals (e.g., noon to night). It appears that before the globalization of time-keeping practices and technologies, humans often used similar gestural practices for this purpose (e.g., Nilsson, 1920). While the practice is unevenly distributed around the globe, it has been independently innovated over and over, and thus ranks as another "natural convention."

\section{Venturing forth: Further recommendations for the future}

The previous two sections have sketched current research on gestural universals and diversity: section 3 crystallized a set of distinctions that can help sharpen our understanding and raise new questions; section 4 surveyed case studies that together demonstrate the constrained gestural diversity we see across communities. Where do we go from here? In what follows I make a few further suggestions for the near future. These "best practices" are already being used in some quarters - but spottily and often implicitly. My aim is to make the case that they should be a more prominent—and more explicit—part of this emerging research enterprise. As the study of gestural universals 
and diversity matures into a self-conscious subfield - perhaps analogous to the subfield of linguistic typology_explicit frameworks, tools, and practices are crucial.

Table 2. Complementary approaches to studying universals and diversity in gesture

\begin{tabular}{|c|c|c|}
\hline approach & example phenomenon & example citation \\
\hline form-centered & head shake & Kendon, 2002 \\
\hline examines the range of & lip-pointing & Sherzer, 1973 \\
\hline functions a form serves & palm-up & Müller, 2004 \\
\hline function-centered & pointing & Wilkins, 2003 \\
\hline $\begin{array}{l}\text { examines the range of forms } \\
\text { that serve a function }\end{array}$ & negation & Mesh \& Hou, 2018 \\
\hline domain-centered & size gestures & not attested \\
\hline examines how gesture & time gestures & not attested \\
\hline expresses a semantic domain & & \\
\hline language-centered & ideophones & Dingemanse, 2013 \\
\hline examines how gesture links to & serial verbs & Defina, 2016 \\
\hline
\end{tabular}

\subsection{Using complementary approaches}

As we have seen, there are several fruitful approaches to uncovering patterns and diversity in gesture (with inspiration from Lucy, 1997). These four approaches reveal different things, and each is valuable (Table 2). A first is centered on form: you take a particular form of interest and examine the variety of ways it is used. Form-centered 
approaches are crucial for getting a sense of the range of meanings that a given form has and, ultimately, for uncovering the structure of these meaning networks - that is, which meanings are core and which are extensions (Figure 2). A second approach is centered on function: you take a communicative function and ask which gestural forms serve that function. Function can be more or less granular. One could consider the wide umbrella of negation, for example, or the narrower umbrellas of refusal, absence, denial, and so on (see, e.g., Beaupoil-Hourdel, Morgenstern, \& Boutet, 2016). Function can also be based, not on semantic notions like negation, but on interactional notions. For instance, one fruitful line of work has examined the linguistic and bodily resources deployed in the course of conversational repair (Dingemanse et al., 2015; Floyd, Manrique, Rossi, \& Torreira, 2016). Function-centered approaches—-however function is defined—are crucial for getting a sense of the gestural repertoires used within a community, as well as for judging questions about which forms are privileged. 


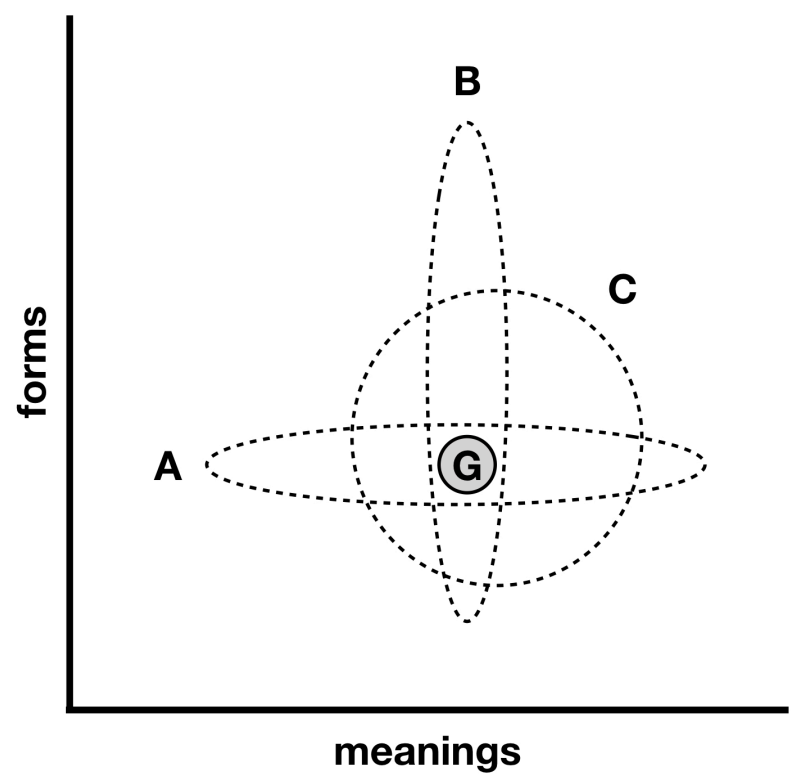

Figure 2. A schematic depiction of different approaches to the study of gesture. Imagine a gesture - that is, a specific form-meaning pairing - marked G. One approach to understanding $\mathrm{G}$ is a form-centered one in which you look at the range of meanings that the form is used for (depicted by the horizontal oval marked A). This approach reveals the meaning networks that $\mathrm{G}$ may participate in. Alternatively, you might want to take a function-centered approach in which you look at the other gestural forms that serve the same or similar function (depicted by the vertical oval marked B). This approach reveals the repertoires that $\mathrm{G}$ may participate in. A third approach is to consider the broader semantic domain in which $\mathrm{G}$ is situated (the circle marked $\mathrm{C}$ ). This approach reveals how different gestures within a broader domain of meanings may cohere. A fourth approach, not pictured, centers on linguistic resources and examines how they link to gesture.

A third approach, closely related to the function-centered approach, is centered on particular semantic domains. Domains that are ripe for this type of inquiry include space (and its subdomains, such as size, motion, shape, and relative location), time (and its subdomains, such as duration, deictic time, sequential time, and time of day), and social structure (including its subdomains of kinship and hierarchy). Zooming out to examine broader meaning domains allows one to see, for example, how the gestural representation of different specific semantic notions (e.g., motion and relative location) may hang together. A fourth approach - not discussed in detail here but well-attested in the gesture 
literature - is centered on linguistic structures. The idea is to look at a particular phenomenon in language and see what gestural regularities, if any, link with it (e.g., Defina, 2016; Dingemanse, 2013; Gullberg \& Narasimhan, 2010; Kita \& Özyürek, 2003). Across the world's languages, there are a number of linguistic phenomena that may prove to be bound up with gesture in interesting ways but have yet to be examined through this lens - examples include switch reference, evidentiality, birth order terms, double tense, and many more.

Each of these approaches has strengths and limitations. An advantage of making the menu of approaches — and our choices among them - more explicit is that it highlights gaps in understanding. As one example, few if any studies have adopted a form-centered approach to index finger extension, which would allow us to tease apart the different ways it is used, whether for pointing or other functions (as, e.g., Sherzer (1973) did for lip-pointing). As another example, I am not aware of any studies that have taken a broad domain-centered approach to time gestures, which would allow for insights into how—or if - the domain of time hangs together in gesture. In sum, my first recommendation for the future is that we keep all these possible approaches in mind when we study gesture, that we use them in a complementary fashion, and that we seek to fill gaps where we see them.

\subsection{Seeking and evaluating explanations}

As we venture forth, we'll be busy with the messy descriptive business of simply characterizing gesture across cultures. But there is another part of our task that is more important: seeking and evaluating explanations for the patterns we find (for discussion of 
related issues in linguistic typology, see, e.g., Haspelmath, 1997). As Kendon (1984) remarked: "From the point of view of the cross-cultural comparison of gesture... the mere description of differences is of little interest. What is needed is a way of inquiring into the reasons for these differences" (p. 108). Description is a first step, but explanation is the end goal.

Good explanations will be multifaceted, reckoning with a range of factors that shape gestural behaviors (see Kita, 2009 for discussion). Some factors push gestural phenomena in the direction of uniformity. For instance, humans are all subject to the same biomechanical constraints — arms that move naturally in some ways but not others, fingers we have fine-grained control over and fingers we don't, and so on. Very basic principles are also at play_-for example, all things being equal, humans favor less effortful movements (e.g. Sanders \& Napoli, 2016). Commonalities can also arise through common cognitive capacities or interactive constraints. The convention of pointing to the sun's arc to refer to time is widespread, but it does not simply fall out of biomechanics. Rather, it is a recurring "solution" to a communicative problem, a solution that leverages a universal cognitive capacity for conceptual mappings along with regularities in the world. And, finally, commonalities can also arise through cultural contact. Gestures are readily borrowable, and are thus often found regionally (e.g., Morris et al., 1979).

Other factors push gestures in the direction of diversity. As Kita (2009) discusses, since gesture so often piggybacks on linguistic structure, linguistic diversity drives gestural diversity; since gestures so often reflect deep cognitive models, cognitive diversity also drives gestural diversity. In particular, differences in favored spatial 
models - whether we prefer to parse space in terms of cardinal directions, bodily axes, or environmental features - lead to broad gestural differences, since such models are used for communicating about both concrete events (e.g., a car crash) and abstract notions (e.g., a "collision" of worldviews). The fact that gestures often reflect a favored spatial model does not imply that they are passive and inert. In fact, gesture may be a key vehicle through which such models are maintained and transmitted in a community (e.g., Le Guen, 2011b). Still other aspects of gestural variation are due to differing communicative ideologies — ideologies that govern whether you should be assertive or meek, whom to respect or avoid, and which uses of the body are untoward. Kendon (1984, 2004) has suggested another candidate factor driving gestural diversity: differences in communicative ecology. He has proposed that this construct might explain why some communities have richer repertoires of conventional gestures than others (Kendon, 2004). Recently, my colleagues and I proposed that communicative ecology could be part of why some groups use more non-manual pointing than others (Cooperrider, Slotta, \& Núñez, 2018).

The trickiest gestures to explain, perhaps, are those for which we simultaneously see both rich diversity and widespread patterns - for example, the case of negation and affirmation. With a nod to Jakobson's phrase about the "interrelation of naturalness and conventionality," I propose that most recurrent gestures and gestural practices are "natural conventions." ${ }^{\prime 3}$ By this phrase I mean that they are culturally selected (i.e., conventionalized) from a menu of motivated (i.e., natural) options (see also the notion of

\footnotetext{
${ }^{3}$ In a related vein, Dingemanse \& Floyd (2014) ask: "Why do similar practices appear in completely unrelated languages? What are the relative roles of natural meaning and conventionalization in the development of these practices?" (p. 446).
} 
"limited possibilities"; Goldenweiser, 1913). For basic communicative functions like negation and pointing, there may be more than one motivated possibility, but groups tend to select one or a few. This proposal has two corollaries. First, there will be very few absolute universals — specific recurrent gestures or gestural practices that are found the world over. But, second, there will be very few one-off cases that are found in one place and only one place. Of course, in many cases explaining why a community selects one motivated form over another will be difficult, but the issue may become tractable as we broaden our base of observations.

Most gesture researchers, of course, do have in mind explanations for the patterns they find, but these explanations sometimes remain implicit and are rarely directly evaluated. An illustration will clarify what I am recommending. In seeking an explanation for the prominence of non-manual pointing in Yupno, we offered a number of possibilities. Each possibility entailed a prediction about where else we might find high rates of non-manual pointing: in Papua New Guinea more broadly; in groups with elaborated demonstrative systems; in small-scale, isolated communities; or in groups who tend to have their hands occupied (Cooperrider, Slotta, \& Núñez, 2018). In a subsequent test of this last "manual availability" explanation, $\mathrm{Li}$ and Cao (2019) compared pointing preferences in two communities within rural China—farmers and herders—-and found that farmers, who report that their hands are more likely to be occupied during their work, also use more non-manual pointing. In sum, my second recommendation is that we make our proposed explanations explicit, so that we-or others—can evaluate them. 


\subsection{Proposing candidate generalizations}

My last recommendation is to propose candidate generalizations (see, e.g., Greenberg, 1978). In the past, gesture researchers have been reluctant to do this. Many papers simply do not comment on whether the phenomena described are proposed to be universal or culture-specific. It is unclear, for example, which uses of the head shake Kendon (2002) thinks might be restricted to Anglo-European contexts and which might be more broadly distributed; we might assume that Enfield et al.'s (2007) observations about pragmatic functions of pointing would generalize beyond rural Laos, but this is not explicitly discussed. Such reticence is perhaps understandable: to venture a generalization is to pin a target to one's chest. But that is precisely the point. Proposing generalizations propels inquiry by formulating clear, tempting targets for future research. To illustrate what I have in mind, I offer a first list of candidate generalizations about the five gestural phenomena discussed earlier (Table 3). Some are quite tentative (e.g., \#3), whereas others are already fairly secure (e.g., \#12.). And, of course, many more could be formulated about these and other aspects of gesture. In my utopic vision for the future of research on gestural universals and diversity, there would be an online resource where all such generalizations are listed, commented on, and updated.

Table 3. Fifteen candidate generalizations about gestural universals and diversity

\begin{tabular}{ll}
\hline phenomenon & generalization \\
\hline negation & 1. All cultures have a repertoire of conventional bodily signals for \\
& negation \\
& 2. All cultures have non-manual signals for negation \\
& 3. In all cultures, non-manual signals for negation are privileged
\end{tabular}


over manual signals

pointing 4. Manual and non-manual pointing are used in all cultures

5. Some cultures prefer non-manual pointing over manual pointing

6. Among manual pointing forms, index finger pointing is privileged in all cultures

palm-up gestures

7. All cultures that use the palm-up epistemic use it to express absence of knowledge

8. All cultures that use the palm-up epistemic also use the shrug to express similar meanings

9. All cultures that use the palm-up epistemic use it in both a prototypical version and in less-effortful, "reduced" forms

size gestures

10. All cultures use gesture to create ad hoc depictions of size

11. All conventional size gestures are iconically motivated

12. Some cultures have conventional gestures associated with spoken language diminutives

time gestures 13. All cultures use gestures to create ad hoc representations of time 14. All cultures use gestures that associate 'now' with the speaker's current location

15. Some cultures use highly conventionalized gestures for specific temporal concepts (e.g., 'yesterday')

\section{Conclusion}

Paraphrasing what the poet Mark Van Doren once observed about human beings, there are two statements about gesture that are true: gesture in all communities is alike and gesture in all communities is different (qtd. in Norenzayan \& Heine, 2005, p. 763). Uncontroversial as both statements are, they have historically been hard to reconcile. This dynamic is hardly unique to the study of gesture, of course. Many of the most contentious debates in the human sciences in recent decades have concerned diversity and universals. Examples include ongoing dust-ups about facial expressions of emotion (e.g., Jack, 
Garrod, Yu, Caldara, \& Schyns, 2012), color terms (e.g., Kay \& Regier, 2003), spatial frames of reference (Majid, Bowerman, Kita, Haun, \& Levinson, 2004), language generally (Evans \& Levinson, 2009), and music (Mehr et al., 2019). In order to make substantive progress on any of these debates, researchers need both better data and better conceptual frameworks. Without these, the debates can circle on, generating much heat and little light. Yet—at least where gesture is concerned-I take the optimistic view that we are poised to make real progress.

And it won't be a moment too soon. Gestural diversity—like so many other aspects of human diversity—is dwindling. On this point, I am more pessimistic. What gestural diversity there is today is no doubt a fraction of the gestural diversity there once was; and, within a century, much of what is left will be gone. The reasons for this rapid loss are several, and they reinforce each other. We are shedding languages and cultures faster than we can mourn them (Evans, 2010). Western ways of conceptualizing the world are ascendant. People are connected like never before and increasingly share a visual culture: it is estimated that Facebook has 2.5 billion active users and that more than half of humanity reportedly watched some portion of the 2018 World Cup ${ }^{4}$. With this new connectedness comes, too, common forms of visual communication. A number of conventional gestures - pointing, the shrug, the thumbs-up, the wave, the eye-roll, and more—are now enshrined in emoji (Gawne \& McCulloch, 2019), and more are being added all the time. Early 2020 saw the introduction of the pinched fingers — or "finger purse"-emoji, which is based on the Italian ma che vuoi gesture. The idea that gesture is

\footnotetext{
${ }^{4}$ Facebook usership estimate from: https://www.statista.com/statistics/264810/numberof-monthly-active-facebook-users-worldwide/ (accessed February, 21, 2020). World Cup viewership estimate from: https://www.fifa.com/worldcup/news/more-than-half-theworld-watched-record-breaking-2018-world-cup (accessed February 21, 2020).
} 
a "universal language" was dubious when Quintillian articulated it two millennia ago, and it remains dubious today. But it may not be dubious for much longer.

Acknowledgments: For insightful comments on earlier versions of this work, I would like to thank Natasha Abner, Mark Dingemanse, Olivier Le Guen, an anonymous reviewer for Gesture, the members of the Goldin-Meadow lab at the University of Chicago, and attendees of the 'Anthropology of Gesture' theme session at ISGS 2018 in Cape Town, South Africa. 


\section{References}

Archer, D. (1997). Unspoken diversity: Cultural differences in gestures. Qualitative Sociology, 20(1), 79-105.

Bavelas, J. B., Chovil, N., Lawrie, D. A., \& Wade, A. (1992). Interactive gestures. Discourse Processes, 15, 469-489.

Beaupoil-Hourdel, P., Morgenstern, A., \& Boutet, D. (2016). A child's multimodal negations from 1 to 4: The interplay of modalities. In P. Larrivée \& C. Lee (Eds., Negation and polarity: Experimental perspectives (pp. 95-123). Cham: Springer.

Best, E. (1924). The Maori: Volume One. Wellington, NZ: H.H. Tombs.

Bonvillian, J. D., Ingram, V. L., \& McCleary, B. M. (2009). Observations on the Use of Manual Signs and Gestures in the Communicative Interactions between Native Americans and Spanish Explorers of North America: The Accounts of Bernal Díaz del Castillo and Álvar Núñez Cabeza de Vaca. Sign Language Studies, 9(2), 132-165.

Bressem, Jana and Cornelia Müller (2014). The family of AWAY-gestures. In C. Müller, A. Cienki, E. Fricke, S. H. Ladewig, D. McNeill \& J. Bressem (Eds.), Body-LanguageCommunication (vol. 2) (pp.1592-1604). Boston: De Gruyter: Mouton.

Brentari, Diane, Giovanna Marotta, Ilaria Margherita, and Angela Ott (2013). The interaction of pitch accent and gesture production in Italian and English. Studi e Saggi Linguistici, 51(1), 79-97.

Bressem, J., Stein, N., \& Wegener, C. (2017). Multimodal language use in Savosavo: Refusing, excluding and negating with speech and gesture. Pragmatics, 27(2), 173-206.

Brookes, H. (2004). A repertoire of South African quotable gestures. Journal of Linguistic Anthropology, 14(2), 186-224.

Bulwer, John (1975 [orig. 1644]). Chirologia: Or the natural language of the hand and Chironomia: Or the art of manual rhetoric. J. W. Cleary (Ed.) Carbondale, IL: Southern Illinois University Press.

Calbris, G. (1990). The Semiotics of French Gestures. Bloomington, IN: Indiana University Press.

Casasanto, D., \& Jasmin, K. (2012). The hands of time: Temporal gesture in English speakers. Cognitive Linguistics, 23(4), 643-674.

Carayon, C. (2016). "The Gesture Speech of Mankind": Old and new entanglements in the histories of American Indian and European sign languages. American Historical Review, 121(2), 461-491. 
Clark, H. H. (1996). Using language. Cambridge: Cambridge University Press.

Clark, H. H. (2003). Pointing and placing. In S. Kita (Ed.), Pointing: Where language, culture, and cognition meet (pp. 243-268). Mahwah, NJ: Lawrence Erlbaum.

Clark, H. H. (2016). Depicting as a method of communication. Psychological review, 123(3), 324.

Cooperrider, K. (2017). Foreground gesture, background gesture. Gesture, 16(3), 176202.

Cooperrider, K. (2018, May 14). From pointing to nodding: Is gesture a universal language? Aeon. Retrieved from: https://aeon.co/essays/from-pointing-to-nodding-isgesture-a-universal-language

Cooperrider, K., \& Núñez, R. (2012). Nose-pointing: Notes on a facial gesture of Papua New Guinea. Gesture, 12(2), 103-129.

Cooperrider, K., Abner, N., \& Goldin-Meadow, S. (2018). The palm-up puzzle: Meanings and origins of a widespread form in gesture and sign. Frontiers in Communication, 3(June), 1-16. http://doi.org/10.3389/fcomm.2018.00023

Cooperrider, K., Slotta, J., \& Núñez, R. (2018). The preference for pointing with the hand is not universal. Cognitive Science, 42(4), 1375-1390.

Cooperrider, K., Núñez, R., \& Sweetser, E. (2014). The conceptualization of time in gesture. In C. Müller, A. Cienki, E. Fricke, S. Ladewig, D. McNeill, \& J. Bressem (Eds.), Body-Language-Communication (vol. 2) (pp. 1781-1788). New York: Mouton de Gruyter.

Darwin, C. (1872). The expression of emotions in man and the animals. London: John Murray.

de Ruiter, J. P., \& Wilkins, D. P. (1998). The synchronization of gesture and speech in Dutch and Arrernte (an Australian Aboriginal language): A cross-cultural comparison. In S. Santi (Ed.), Oralité et gestualité (pp. 603-607). Paris: Harmattan.

Defina, R. (2016). Do serial verb constructions describe single events? A study of cospeech gestures in Avatime. Language, 92(4), 890-910

Dégerando, J.-M. (1969 [orig. 1800]). The observation of savage peoples [trans. F. C. T. Moore]. London: Routledge.

Dingemanse, M. (2013). Ideophones and gesture in everyday speech. Gesture, 13(2), 143-165. 
Dingemanse, M., \& Floyd, S. (2014). Conversation across cultures. In N. J. Enfield, P. Kockelman, \& J. Sidnell (Eds.), The Cambridge Handbook of Linguistic Anthropology (pp. 447-480). Cambridge: Cambridge University Press.

Dingemanse, M., Roberts, S. G., Baranova, J., Blythe, J., Drew, P., Floyd, S., ... Enfield, N. J. (2015). Universal principles in the repair of communication problems. Plos One, 10(9), e0136100. http://doi.org/10.1371/journal.pone.0136100

Eco, U. (1976). A theory of semiotics. Bloomington: Indiana University Press.

Efron, D. (1941). Gesture and environment. New York: Kings Crown Press.

Enfield, N. J. (2001). 'Lip-pointing': A discussion of form and function with reference to data from Laos. Gesture, 1(2), 185-211.

Enfield, N. J. (2009). The anatomy of meaning. Cambridge: Cambridge University Press.

Enfield, N. J., Kita, S., \& de Ruiter, J. P. (2007). Primary and secondary pragmatic functions of pointing gestures. Journal of Pragmatics, 39(10), 1722-1741.

Engelke, M. (2018). How to think like an anthropologist. Princeton, NJ: Princeton University Press.

Evans, N. (2010). Dying words: Endangered languages and what they have to tell us. London: Wiley-Blackwell.

Evans, N., \& Levinson, S. C. (2009). The myth of language universals: Language diversity and its importance for cognitive science. Behavioral and Brain Sciences, 32(5), 429-448.

Everett, D. L. (2005). Cultural constraints on grammar and cognition in Pirahã: Another look at the design features of human language. Current Anthropology, 46(4), 621-646.

Fox Tree, E. (2009). Meemul Tziij: An indigenous sign language complex of Mesoamerica. Sign Language Studies, 9(3), 324-366.

Floyd, S. (2016). Modally hybrid grammar? Celestial pointing for time-of-day reference in Nheengatú. Language, 92(1), 31-64.

Floyd, S., Manrique, E., Rossi, G., \& Torreira, F. (2016). Timing of visual bodily behavior in repair sequences: Evidence from three languages. Discourse Processes, 53(3), 175-204.

Gawne, L. (2018), Contexts of use of a rotated palms gesture among Syuba (Kagate) speakers in Nepal. Gesture 17(1), 37-64. 
Gawne, L., \& McCulloch, G. (2019). Emoji as digital gestures. Language@Internet, 17, article 2 .

Goldenweiser, A. A. (1913). The principle of limited possibilities in the development of culture. The Journal of American Folklore, 26(101), 259-290.

Greenberg, Joseph. 1978. Generalizations about numeral systems. In J. Greenberg, C. A., Ferguson, \& E. A. Moravcsik (Eds.), Universals of Human Language, vol. 3 (pp. 249295). Stanford: Stanford University Press.

Gu, Y., Zheng, Y., \& Swerts, M. (2019). Having a different pointing of view about the future: The effect of signs on temporal gestures in Mandarin-CSL bimodal bilinguals. Bilingualism: Language and Cognition. 22(4), 836-847.

Gullberg, M., \& Narasimhan, B. (2010). What gestures reveal about the development of semantic distinctions in Dutch children's placement verbs. Cognitive Linguistics, 21(2), 239-262.

Harrison, S. (2014). The organisation of kinesic ensembles associated with negation. Gesture, 14(2), 117-140.

Haspelmath, M. (1997). Indefinite pronouns. Oxford: Clarendon Press.

Healy, C. (2012). Pointing to show agreement. Semiotica, 192, 175-195.

Hewes, G. W. (1974). Gesture language in culture contact. Sign Language Studies, 4(1), $1-34$.

Huffman, R. (1931). Nuer customs and folklore. London: International Institute of African Language and Culture.

Inbar, A., \& Shor, L. (2019). Covert negation in Israeli Hebrew: Evidence from cospeech gestures. Journal of Pragmatics, 143, 85-95.

Jack, R. E., Garrod, O. G. B., Yu, H., Caldara, R., \& Schyns, P. G. (2012). Facial expressions of emotion are not culturally universal. Proceedings of the National Academy of Sciences, 109(19), 7241-7244.

Jakobson, R. (1972). Motor signs for “yes" and "no.” Language in Society, 1(1), 91-96.

Jehoul, A., Brône, G., \& Feyaerts, K. (2017). The shrug as marker ofobviousness. Linguistics Vanguard 3:20160082. doi: 10.1515/lingvan-2016-0082

Johnson, H. G., Ekman, P., \& Friesen, W. V. (1975). Communicative body movements: American emblems. Semiotica, 15(4), 335-353. 
Jurafsky, D. (1996). Universal tendencies in the semantics of the diminutive. Language, $72(3), 533-578$.

Kay, P., \& Regier, T. (2003). Resolving the question of color naming universals. Proceedings of the National Academy of Sciences, 100(15), 9085-9089.

Kendon, A. (1984). Did gesture have the happiness to escape the curse at the confusion of Babel? In A. Wolfgang (Ed.), Nonverbal behavior: perspectives, applications, intercultural insights (pp. 75-114). Lewiston, NY: Hogrefe.

Kendon, A. (1988). Sign languages of Aboriginal Australia: Cultural, semiotic and communicative perspectives. Cambridge: Cambridge University Press.

Kendon, A. (2002). Some uses of the head shake. Gesture, 2(2), 147-182.

Kendon, A. (2004). Gesture: Visible action as utterance. Cambridge: Cambridge University Press.

Kita, S. (2009). Cross-cultural variation of speech-accompanying gesture: A review. Language and Cognitive Processes, 24(2), 145-167.

Kita, S., \& Essegbey, J. (2001). Pointing left in Ghana How a taboo on the use of the left hand influences gestural practice. Gesture, 1(1), 73-95.

Kita, S., \& Özyürek, A. (2003). What does cross-linguistic variation in semantic coordination of speech and gesture reveal?: Evidence for an interface representation of spatial thinking and speaking. Journal of Memory and Language, 48(1), 16-32.

Knowlson, J. R. (1965). The idea of gesture as a universal language in the XVIIth and XVIIIth centuries. Journal of the History of Ideas, 26(4), 495-508.

Knox, D. (1990). Ideas on gesture and universal languages, c.1550-1650. In New perspectives on Renaissance thought: Essays in the history of science, education and philosophy: in memory of Charles B. Schmitt (pp. 101-136). London: Duckworth.

LaBarre, W. (1947). The cultural basis of emotions and gestures. Journal of Personality, 16(1), 49-68.

Ladewig, S. H. (2011). Putting the cyclic gesture on a cognitive basis. CogniTextes. Revue de l'Association française de linguistique cognitive, 6. DOI :

10.4000/cognitextes.406

Ladewig, Silva H. (2014). Recurrent gestures. In C. Müller, A. Cienki, E. Fricke, S. Ladewig, D. McNeill \& J. Bressem (Eds.), Body-Language-Communication: An 
international handbook on multimodality in human interaction (pp. 1558-1575). Berlin: De Gruyter: Mouton.

Le Guen, O. (2011a). Modes of pointing to existing spaces and the use of frames of reference. Gesture, 11(3), 271-307.

Le Guen, O. (2011b). Speech and gesture in spatial language and cognition among the Yucatec Mayas. Cognitive Science, 35, 905-938.

Le Guen, O., \& Balam, L. I. P. (2012). No Metaphorical Timeline in Gesture and Cognition Among Yucatec Mayas. Frontiers in Psychology, 3(August), 1-15. http://doi.org/10.3389/fpsyg.2012.00271

Le Guen, O., Petatillo, R., \& Canché, R. (in press). Yucatec Maya multimodal interaction as basis for Yucatec Maya Sign Language.

Levinson, S. C., \& Majid, A. (2013). The island of time: Yélî Dnye, the language of Rossel Island. Frontiers in Psychology, 4(February), 61. https://doi.org/10.3389/fpsyg.2013.00061

Li, H., \& Cao, Y. (2019). Hands occupied: Chinese farmers use more non-manual pointing than herders. Lingua. https://doi.org/10.1016/j.lingua.2019.02.006

Liszkowski, U., Brown, P., Callaghan, T., Takada, A., \& de Vos, C. (2012). A prelinguistic gestural universal of human communication. Cognitive Science, 36, 698713.

Lucy, J. A. (1997). Linguistic relativity. Annual Review of Anthroplogy, 26, 291-312.

Majid, A., Bowerman, M., Kita, S., Haun, D. B. M., \& Levinson, S. C. (2004). Can language restructure cognition? The case for space. Trends in Cognitive Sciences, 8(3), $108-114$.

Mallery, G. (1882). The gesture speech of man. American Annals of the Deaf and Dumb, $27(2), 69-89$.

Mandel, M. (1977). Iconic devices in American Sign Language. In L. Friedman (Ed.), On the other hand: New perspectives on American Sign Language (pp. 57-107). New York: Academic Press.

Mauss, M. (1973 [orig. 1935]). Techniques of the body. Economy and Society, 2(1), 7088.

McNeill, D. (1992). Hand and mind. Chicago: Chicago University Press. 
McClave, E. (2007). Potential cognitive universals: Evidence from head movements in Turkana. In S. Duncan, J. Cassell, \& E. T. Levy (Eds.), Gesture and the Dynamic Dimension of Language. Essays in honor of David McNeill (pp. 91-98). Philadelphia: John Benjamins.

McClave, E., Kim, H., Tamer, R., \& Mileff, M. (2007). Head movements in the context of speech in Arabic, Bulgarian, Korean, and African-American Vernacular English. Gesture, 7(3), 343-390.

Mehr, S. A., Singh, M., Knox, D., Ketter, D. M., Pickens-Jones, D., Atwood, S., ... Glowacki, L. (2019). Universality and diversity in human song. Science, 366(November, eaax0868), 1-17. https://doi.org/10.1126/science.aax0868

Mesh, K. (2017). Points of comparison: What indicating gestures tell us about the origins of signs in San Juan Quiahije Chatino Sign Language. (Doctoral dissertation: The University of Texas at Austin.)

Mesh, K. \& Hou, L. (2018). Negation in San Juan Quiahije Chatino Sign Language: The integration and adaptation of negative emblems. Gesture, 17(3), 330-374.

Morris, D., Collett, P., Marsh, P., \& O'Schaughnessy, M. (1979). Gestures: Their origins and distribution. London: Jonathan Cape.

Müller, C. (2004). Forms and uses of the palm up open hand: A case of a gesture family? In C. Müller \& R. Posner (Eds.), The semantics and pragmatics of everyday gestures (pp. 233-256). Berlin: Weidler.

Müller, C. (2017). How recurrent gestures mean: Contextualized contexts-of-use and embodied motivation. Gesture, 16(2), 276-303.

Nilsson, N. (1920). Primitive time-reckoning. Lund, Sweden: C. W. K. Gleerup.

Nobe, S. (2000). Where do most spontaneous representational gestures actually occur with respect to speech? In D. McNeill (Ed.), Language and gesture (pp. 186-198). Cambridge: Cambridge University Press.

Norenzayan, A., \& Heine, S. J. (2005). Psychological universals: What are they and how can we know? Psychological Bulletin, 131(5), 763-84.

Núñez, R. E., \& Sweetser, E. (2006). With the future behind them: Convergent evidence from Aymara language and gesture in the crosslinguistic comparison of spatial construals of time. Cognitive Science, 30(3), 401-450.

Nyst, V. (2016). Size and shape depictions in the manual modality: A taxonomy of iconic devices in Adamorobe Sign Language. Semiotica, 210, 75-104. 
Quintilianus, Marcus Fabius (1922 [orig. 95 C.E.]). The Institutio Oratoria of Quintilian. Translated by Harold Edgeworth Butler, Volume IV, The Loeb Classical Library. New York: G. P. Putnam and Sons.

Pouw, W., Harrison, S. J. \& Dixon, J. A. (2019). Gesture-speech physics: The biomechanical basis for the emergence of gesture-speech synchrony. Journal of Experimental Psychology: General. doi: 10.1037/xge0000646

Roseano, P., González, M., Borràs-Comes, J., \& Prieto, P. (2014). Communicating epistemic stance: How speech and gesture patterns reflect epistemicity and evidentiality. Discourse Processes, 53(3), 135-174.

Sanders, N., \& Napoli, D. J. (2016). Reactive effort as a factor that shapes sign language lexicons. Language, 92(2), 275-297.

Sapir, E. (1927/1949). The unconscious patterning of behavior in society. In D.G. Mandelbaum (Ed.), Selected writings of Edward Sapir (pp. 544-559). Berkeley, California: University of California Press.

Schegloff, E. A. (1984). On some gestures' relation to talk. In J. M. Atkinson \& J. Heritage (Eds.), Structures of Social Action (pp. 266-298). Cambridge: Cambridge University Press.

Sherzer, J. (1973). Verbal and nonverbal deixis: The pointed lip gesture among the San Blas Cuna. Language in Society, 2, 117-131

Sinha, C., Sinha, V. D. S., Zinken, J., \& Sampaio, W. (2011). When time is not space: The social and linguistic construction of time intervals and temporal event relations in an Amazonian culture. Language and Cognition, 3(1), 137-169.

Stocking, G. W. (1995). Delimiting anthropology: Historical reflections on the boundaries of a boundless discipline. Social Research, 62(4), 932-966. https://doi.org/Article

Sullivan, K., \& Bui, L. T. (2016). With the future coming up behind them: Evidence that time approaches from behind in Vietnamese. Cognitive Linguistics, 27(2), 205-233.

Sweetser, E. (1990). From etymology to pragmatics: Metaphorical and cultural aspects of semantics. Cambridge: Cambridge University Press.

Tylor, E. (1878). Researches into the early history of mankind. London: John Murray.

Vandenabeele, B. (2002). No need for essences. On non-verbal communication in first inter-cultural contacts. South African Journal of Philosophy, 21(2), 85-96. 
Walker, E., \& Cooperrider, K. (2016). The continuity of metaphor: Evidence from temporal gestures. Cognitive Science, 40, 481-495.

Wilkins, D. (2003). Why pointing with the index finger is not a universal (in sociocultural and semiotic terms). In S. Kita (Ed.), Pointing: Where language, culture, and cognition meet (pp. 171-215). Mahwah, NJ: Laurence Erlbaum. 